\title{
An Improved User Authentication Scheme for Hierarchical Wireless Sensor Networks without Tamper-Proof Smart Card
}

\author{
Min-Shiang Hwang \\ Depart. of Computer Science \& Information Engineering \\ Asia University \\ Taichung, Taiwan \\ Email: mshwang@asia.edu.tw \\ Yuen-Cheng Chou \\ Depart. of Computer Science \& Information Engineering \\ Asia University \\ Taichung, Taiwan
}

\author{
Eko Fajar Cahyadi \\ Depart. of Computer Science \& Information Engineering \\ Asia University \\ Taichung, Taiwan
}

\author{
Cheng-Ying Yang \\ Department of Computer Science \\ University of Taipei \\ Taipei, Taiwan
}

\begin{abstract}
Recently, Maitra et al. proposed an efficient and robust user authentication scheme for hierarchical wireless sensor networks. They claimed that their scheme does not need tamper-proof smart card and resisted different possible attacks include smart card stolen attack, impersonation attack, privileged insider attack, replay attack, off-line password guessing attack, theft attack, session key recovery attack, denial of service attack, and cluster head capture attack. However, we find some weaknesses of his scheme in this article. We show that their scheme is vulnerable to off-line password guessing with smart card stolen attack.
\end{abstract}

Keywords-password; smart card; tamper-proof; user authentication; wireless sensor networks;

\section{INTRODUCTION}

Remote user authentication is the mechanism that widely uses to identify the legitimate user in Internet [8], [12], [21], [27], [28]. Conventional remote user authentication schemes are suited to identify the remote users for single server environment of client/server architecture [2], [7], [17], [20], [24], [26]. However, the use of Internet has grown spectacularly. More and more users need services in different servers. In other word, the users in the network architecture are become in multi-server environment [4], [6], [10], [15]. In conventional user authentication schemes, users not only need to login to various servers with repetitive registration, but also need to remember the various user ID (identities) and passwords [3], [5], [9], [11].

In 2012, Ramasamy et al. proposed a user authentication scheme for smart cards [19]. However, Thandra et al. showed that their scheme is insecure [22]. In 2016, Thandra et al. also proposed a secure and efficient user authentication scheme [22]. However, Pan et al. shown that their scheme is vulnerable to denial of service, online and offline password guessing, and user impersonation attacks [18]. In 2016, Wei et al. proposed a user authentication scheme [25].
However, Tsai et al. shown that their scheme is vulnerable to denial of service, password guessing, and privileged insider attacks [23]. Recently, Liu et al. proposed an efficient user authentication scheme with a smart card [14]. However, Liu et al. shown that their scheme was vulnerable to the replaying attack [13].

Recently, Maitra et al. proposed an efficient and robust user authentication scheme for hierarchical wireless sensor networks [16]. They claimed that their scheme does not need tamper-proof smart card and resisted different possible attacks include smart card stolen attack, impersonation attack, privileged insider attack, replay attack, off-line password guessing attack, theft attack, session key recovery attack, denial of service attack, and cluster head capture attack. However, we find some weaknesses of his scheme in this article. We show that their scheme is vulnerable to off-line password guessing with smart card stolen attack and off-line password guessing with smart card stolen attack.

The rest of this paper is organized as follows. In Section 2, we briefly review Maitra et al.'s remote user authentication scheme. In Section 3, we analyze and show that some security flaws exist in Maitra et al.'s user authentication scheme. Finally, we present our conclusions in Section 4.

\section{REVIEW OF MAitRA ET AL.'s SCHEME}

In this section, we briefly review Maitra et al.'s user authentication scheme for hierarchical wireless sensor networks without tamper-proof smart card [16]. There are four participants in Maitra et al.'s remote user authentication scheme: users $\left(U_{i}, i=1,2, \cdots, m\right.$ for short); Card reader (CR for short); Base stations (BS for short) and cluster head $\left(C H_{j}, j=1,2, \cdots, n\right.$ for short). The scheme consists of four phases, namely the registration phase, the login phase, the authentication phase, and the password change phase. The notations used in this article are listed in Table ??. 
Table I

LIST OF NOTATION USED

\begin{tabular}{|r|l|}
\hline \hline Symbol & Description \\
\hline$U_{i}$ & $i$-th User \\
\hline BS & Base station \\
\hline$S N_{j}$ & Sensor node $j$ \\
\hline$C H_{j}$ & Cluster head $j$ in the $j$-th cluster \\
\hline$p w_{i}$ & Password of user $U_{i}$ \\
\hline$I D_{i}$ & Identity of user $U_{i}$ \\
\hline$I D_{C H_{j}}$ & Identity of cluster head $C H_{j}$ \\
\hline$I D_{S N_{j}}$ & Identity of sensor node $S N_{j}$ \\
\hline$S_{C H_{j}}$ & Shared secret key between $C H_{j}$ and $B S$ \\
\hline$S_{S N_{j}}$ & Shared secret key between $S N_{j}$ and $B S$ \\
\hline$M K_{C H_{j}}$ & Unique shared master key randomly \\
\hline$S K$ & Senerated by the BS for $C H_{j}$ \\
\hline$h(\cdot)$ & Cryptographic One-way hash function \\
\hline$E$ & Symmetric key encryption algorithm \\
\hline$D$ & Symmetric key decryption algorithm \\
\hline$s$ & Secret information of the base station \\
\hline$X_{A}$ & Shared secret between $U_{i}$ and $B S$ \\
\hline$T$ & Current time stamp \\
\hline$\|$ & Concatenation operation \\
\hline$\oplus$ & Bit wise XOR operation \\
\hline
\end{tabular}

\section{A. The Registration Phase}

In the registration phase, the base station $B S$ makes a smart card for a new user $\left(U_{i}\right)$. The registration phase is executed as follows:

1) The new user $U_{i}$ firstly chooses a random number $y_{i}$, his/her identity $I D_{i}$ and password $p w_{i}$.

2) $U_{i}$ computes $p w r_{i}=h\left(\begin{array}{lll}p w_{i} & \| & y_{i}\end{array}\right)$ and sends $\left\{I D_{i}, p w r_{i}\right\}$ to the base station $B S$ through a secure channel.

3) After getting message $\left\{I D_{i}, p w r_{i}\right\}$ from the user $U_{i}$, base station computes $X_{i}=h\left(I D_{i} \| s\right) \oplus p w r_{i}$ and $B_{i}=h\left(h\left(I D_{i} \| s\right) \| p w r_{i}\right)$.

4) The base station issues a smart card for user $U_{i}$ by storing $\left\{X_{i}, B_{i}, h(\cdot)\right\}$ into the memory of smart card.

5) After getting his/her smart card, user $U_{i}$ stores $y_{i}$ into the memory of smart card.

\section{B. The Login Phase}

In this phase, the user $\left(U_{i}\right)$ wants to login to the base station $B S_{j}$ for obtaining some services, the user $\left(U_{i}\right)$ firstly attaches his/her smart card to a device reader and inputs his/her identity $I D_{i}^{\prime}$ and password $P W_{i}^{\prime}$. The login phase is executed in the following:

1) Then card reader computes

$$
\begin{aligned}
p w r_{i}^{\prime} & =h\left(p w_{i} \| y_{i}\right), \\
Y_{i}^{\prime} & =X_{i} \oplus p w r_{i}^{\prime}, \\
B_{i}^{\prime} & =h\left(Y_{i}^{\prime} \| p w r_{i}^{\prime}\right),
\end{aligned}
$$

and checks whether computed $B_{i}^{\prime}$ equals stored $B_{i}$. If true, proceed to next otherwise 'rejects' user $U_{i}$.
Then, user $U_{i}$ chooses $I D_{C H_{j}}$ and submits it to the card reader.

2) The card reader further chooses a random number $N_{1}$ and computes

$$
\begin{aligned}
P_{i} & =h\left(Y_{i}^{\prime}\left\|I D_{C H_{j}}\right\| N_{1} \| p w r_{i}^{\prime}\right) \\
R_{i} & =N_{1} \oplus p w r_{i}^{\prime},
\end{aligned}
$$

and sends $\left\{I D_{i}, I D_{C H_{j}}, P_{i}, R_{i}, X_{i}\right\}$ to the base station.

\section{The Authentication Phase}

Upon receiving the authentication request message $\left\{I D_{i}, I D_{C H_{j}}, P_{i}, R_{i}, X_{i}\right\}$ from user $U_{i}$, the base station $B S$ executes this authentication phase in the following:

1) The base station computes

$$
\begin{aligned}
Y_{i}^{*} & =h\left(I D_{i} \| s\right), \\
p w r_{i}^{*} & =Y_{i}^{*} \oplus X_{i}, \\
N_{1}^{*} & =p w r_{i}^{*} \oplus R_{i} \\
P_{i}^{*} & =h\left(Y_{i}^{*}\left\|I D_{C H_{j}}\right\| N_{1}^{*} \| p w r_{i}^{*}\right) .
\end{aligned}
$$

2) $B S$ checks whether computed $P_{i}^{*}$ equals sending $P_{i}$ or not. If it holds good, base station further chooses a random number $N_{2}$ and computes

$$
\begin{aligned}
Z_{i} & =p w r_{i}^{*} \oplus N_{2}, \\
D_{i} & =h\left(Y_{i}^{*}\left\|N_{2}\right\| I D_{C H_{j}}\left\|I D_{i}\right\| N_{1}^{*}\right) .
\end{aligned}
$$

3) $B S$ sends $\left\{I D_{i}, I D_{C H_{j}}, Z_{i}, D_{i}\right\}$ to the user $U_{i}$. Again base station computes

$$
\begin{aligned}
N_{3} & =N_{2} \oplus N_{1}^{*}, \\
V_{i} & =h\left(I D_{C H_{j}} \| S_{C H_{j}}\right), \\
E_{i} & =V_{i} \oplus N_{3} \\
A_{i} & =h\left(Y_{i}^{*}\left\|N_{3}\right\| p w r_{i}^{*}\right), \\
L_{i} & =A_{i} \oplus V_{i} \\
G_{i} & =h\left(S_{C H_{j}}\left\|N_{3}\right\| A_{i}\left\|I D_{i}\right\| I D_{C H_{j}}\right)
\end{aligned}
$$

4) $B S$ sends $\left\{E_{i}, L_{i}, G_{i}, I D_{i}, I D_{C H_{j}}\right\}$ to the cluster head $\mathrm{CH}_{j}$. After that the following computations are performed:

a) After getting reply message $\left\{I D_{i}, I D_{C H_{j}}, Z_{i}, D_{i}\right\}$ from base station, card reader computes $N_{2}^{\prime}=Z_{i} \oplus p w r_{i}^{\prime}, D_{i}^{\prime}=$ $h\left(Y_{i}^{\prime}\left\|N_{2}^{\prime}\right\| I D_{C H_{j}}\left\|I D_{i}\right\| N_{1}\right)$ and checks whether computed $D_{i}^{\prime}$ equals sending $D_{i}$ or not. If it holds good then computes $N_{3}^{\prime}=N_{1} \oplus N_{2}^{\prime}$, $A_{i}^{\prime}=h\left(Y_{i}^{\prime}\left\|N_{3}^{\prime}\right\| p w r_{i}^{\prime}\right)$ and session key $S K=$ $h\left(I D_{i}\left\|I D_{C H_{j}}\right\| N_{3}^{\prime} \| A_{i}^{\prime}\right)$.

b) After receiving message $\left\{E_{i}, L_{i}, G_{i}, I D_{i}, I D_{C H_{j}}\right\} \quad$ from base station, cluster head $\mathrm{CH}_{j}$ computes $V_{i}^{\star}=$ $h\left(I D_{C H_{j}} \| S_{C H_{j}}\right), N_{3}^{\star}=V_{i}^{\star} \oplus E_{i}, A_{i}^{\star}=L_{i} \oplus V_{i}^{\star}$ and $G_{i}^{\star}=h\left(S_{C H_{j}}\left\|N_{3}^{\star}\right\| A_{i}^{\star}\left\|I D_{i}\right\| I D_{C H_{j}}\right)$ 
and checks weather computed $G_{i}^{\star}$ equals sending $G_{i}$ or not. If true, then it computes session key $S K=h\left(I D_{i}\left\|I D_{C H_{j}}\right\| N_{3}^{\star} \| A_{i}^{\star}\right)$.

Now, both parties (user $U_{i}$ and cluster head $\mathrm{CH}_{j}$ ) are agreed with common shared session key $S K$ and can communicate securely to each other by shared secret session key $S K$ in future.

\section{CRYPtAnAlysis Of MAitra ET AL.'s SCHEME}

In this section, we will analyze Maitra et al.'s user authentication scheme for hierarchical wireless sensor networks without tamper-proof smart card [16]. Maitra et al. claimed that their scheme is resisted different possible attacks include smart card stolen attack, impersonation attack, privileged insider attack, replay attack, off-line password guessing attack, theft attack, session key recovery attack, denial of service attack, and cluster head capture attack. In this section, we show that Maitra et al.'s user authentication scheme is vulnerable to off-line password guessing with smart card stolen attack.

\section{A. Off-line Password Guessing with Smart Card Stolen Attack}

Maitra et al. claimed that an attacker is hard to derive user's password $P W_{i}$ if the attacker gets the user's smart card and a login message $\left\{I D_{i}, I D_{C H_{j}}, P_{i}, R_{i}, X_{i}\right\}$ between the user $U_{i}$ and base station $B S$. In this section, we will show that Maitra et al.'s scheme is vulnerable to off-line password guessing with smart card stolen attack.

The attacker is able to intercept from the public channel. Thus the attacker obtains a login message $\left\{I D_{i}, I D_{C H_{j}}, P_{i}, R_{i}, X_{i}\right\}$ between the user $U_{i}$ and base station $B S$. The attacker may guess the user's password $P W_{i}$ as follows.

1) The attacker guesses the user's password $P W^{\prime}$.

2) The smart card computes $p w r_{i}^{\prime}$ as follows:

$$
p w r_{i}^{\prime}=h\left(P W^{\prime} \| y_{i}\right),
$$

here $y_{i}$ is obtained from the smart card.

3) The smart card computes $Y_{i}^{\prime}$ and $N_{1}^{\prime}$ as follows:

$$
\begin{aligned}
Y_{i}^{\prime} & =X_{i} \oplus p w r_{i}^{\prime}, \\
N_{1}^{\prime} & =R_{i} \oplus p w r_{i}^{\prime} .
\end{aligned}
$$

Here, $X_{i}$ and $R_{i}$ are intercepted from the last login message between the smart card and base station.

4) The attacker computes $P_{i}^{\prime}$ as follows:

$$
P_{i}^{\prime}=h\left(Y_{i}^{\prime}\left\|I D_{C H_{j}}\right\| N_{1}^{\prime} \| p w r_{i}^{\prime}\right) .
$$

Next the attacker checks if $P_{i}^{\prime}$ is or not equal to $P_{i}$, here $P_{i}$ is intercepted from the last login message between the smart card and base station. If it's hold, the guessed password is correct, otherwise, the attacker guess other password and checks it again as the above steps.

The attacker could repeat the above step to re-guess the other password. If it is true, this implies which the guessing password $P W_{i}^{\prime}$ is correct. Therefore, Maitra et al.'s user authentication scheme is vulnerable to the off-line password guessing with smart card stolen attack.

\section{B. The improvement of Maitra et al.'s's Scheme}

The main weakness of Maitra et al.'s's user authentication scheme is that the attacker could repeat to guess the password with smart card. To improve the weakness of Maitra et al.'s's scheme, the smart card in this scheme should set up the timer. If the user input the incorrect password 3 times, the smart card must initial the registration of the user.

\section{CONCLUSION}

In this article, we have reviewed Maitra et al.'s user authentication scheme for hierarchical wireless sensor networks without tamper-proof smart card [16] and cryptanalyzed its security. Because the user password is chosen by easy to remember, we showed that Maitra et al.'s user authentication scheme cannot withstand the off-line password guessing with smart card stolen attack. We also propose an improvement of Maitra et al.'s's Scheme in this article.

\section{ACKNOWLEDGMENT}

This study was supported by the National Science Council of Taiwan under grant MOST 104-2221-E-468-004.

\section{REFERENCES}

[1] R. Amin, "Cryptanalysis and Efficient Dynamic ID Based Remote User Authentication Scheme in Multi-server Environment Using Smart Card", International Journal of Network Security, Vol. 18, No. 1, pp. 172-181, 2016.

[2] N. Anwar, I. Riadi, A. Luthfi, " Forensic SIM Card Cloning Using Authentication Algorithm", International Journal of Electronics and Information Engineering, Vol. 4, No. 2, pp. 71-81, 2016.

[3] C. C. Chang, W. Y. Hsueh, T. F. Cheng, "An Advanced Anonymous and Biometrics-based Multi-server Authentication Scheme Using Smart Cards", International Journal of Network Security, Vol. 18, No. 6, pp. 1010-1021, 2016.

[4] T. Y. Chen, C. C. Lee, M. S. Hwang, J. K. Jan, "Towards Secure and Efficient User Authentication Scheme Using Smart Card for Multi-Server Environments", The Journal of Supercomputing, Vol. 66, No. 2, pp. 1008-1032, 2013.

[5] T. H. Feng, C. H. Ling, and M. S. Hwang, "Cryptanalysis of Tan's Improvement on a Password Authentication Scheme for Multi-server Environments", International Journal of Network Security, Vol. 16, No. 4, pp. 318-321, 2014. 
[6] D. He, W. Zhao, and S. Wu, "Security Analysis of a Dynamic ID-based Authentication Scheme for Multi-server Environment Using Smart Cards", International Journal of Network Security, Vol. 15, No. 5, pp. 350-356, 2013.

[7] M. S. Hwang, L. H. Li, ”A New Remote User Authentication Scheme Using Smart Cards", IEEE Transactions on Consumer Electronics, Vol. 46, No. 1, pp. 28-30, 2000.

[8] C. C. Lee, M. S. Hwang, I. E. Liao, "Security Enhancement on a New Authentication Scheme with Anonymity For Wireless Environments", IEEE Transactions on Industrial Electronics, Vol. 53, No. 5, pp. 1683-1687, 2006.

[9] L. H. Li, I. C. Lin, M. S. Hwang, "A Remote Password Authentication Scheme for Multi-server Architecture Using Neural Networks", IEEE Transactions on Neural Networks, Vol. 12, pp. 1498-1504, 2001.

[10] I. C. Lin, M. S. Hwang, L. H. Li, "A New Remote User Authentication Scheme for Multi-Server Architecture", Future Generation Computer Systems, vol. 19, no. 1, pp. 13-22, 2003.

[11] C. H. Ling, W. Y. Chao, S. M. Chen, and M. S. Hwang, "Cryptanalysis of Dynamic Identity Based on a Remote User Authentication Scheme for a Multi-server Environment", in 2015 International Conference on Advances in Mechanical Engineering and Industrial Informatics (AMEII 2015), Zhengzhou, April 11-12, 2015, Advances in Engineering Research, vol. 15, pp. 981-986, Atlantis Press, 2015.

[12] J. Ling, G. Zhao, "An Improved Anonymous Password Authentication Scheme Using Nonce and Bilinear Pairings", International Journal of Network Security, Vol. 17, No. 6, pp. 787-794, 2015.

[13] C. W. Liu, C. Y. Tsai, and M. S. Hwang, "Cryptanalysis of an Efficient and Secure Smart Card Based Password Authentication Scheme", Recent Developments in Intelligent Systems and Interactive Applications, Lecture Notes in Computer Science, Springer, 2017.

[14] Y. Liu, C. C. Chang, S. C. Chang, "An Efficient and Secure Smart Card Based Password Authentication Scheme", International Journal of Network Security, Vol. 19, No. 1, pp. 1-10, 2017.

[15] Y. Liu, C. C. Chang, C. Y. Sun, "Notes on An Anonymous Multi-server Authenticated Key Agreement Scheme Based on Trust Computing Using Smart Card and Biometrics", International Journal of Network Security, Vol. 18, No. 5, pp. 9971000, 2016.

[16] T. Maitra, R. Amin, D. Giri, and P. D. Srivastava, "An Efficient and Robust User Authentication Scheme for Hierarchical Wireless Sensor Networks without Tamper-Proof Smart Card", International Journal of Network Security, Vol. 18, No. 3, pp. 553-564, 2016

[17] E. O. Osei, J. B. Hayfron-Acquah, "Cloud Computing Login Authentication Redesign", International Journal of Electronics and Information Engineering, Vol. 1, No. 1, pp. 1-8, 2014.

[18] Chiu-Shu Pan, Cheng-Yi Tsai, Shyh-Chang Tsaur, MinShiang Hwang, "Cryptanalysis of an Efficient Password Authentication Scheme", 2016 3rd International Conference on Systems and Informatics (ICSAI 2016), 2016.
[19] R. Ramasamy and A. P. Muniyandi, "An Efficient Password Authentication Scheme for Smart Card", International Journal of Network Security, Vol. 14, No. 3, pp. 180-186, 2012.

[20] J. J. Shen, C. W. Lin, M. S. Hwang, "A Modified Remote User Authentication Scheme Using Smart Cards", IEEE Transactions on Consumer Electronics, Vol. 49, No. 2, pp. 414-416, 2003.

[21] M. Stanek, "Weaknesses of Password Authentication Scheme Based on Geometric Hashing", International Journal of Network Security, Vol. 18, No. 4, pp. 798-801, 2016.

[22] P. K. Thandra, J. Rajan, and S. A. V. S. Murty, ”Cryptanalysis of an Efficient Password Authentication Scheme", International Journal of Network Security, Vol. 18, No. 2, pp. 362-368, 2016.

[23] C. Y. Tsai, C. S. Pan, and M. S. Hwang, "An Improved Password Authentication Scheme for Smart Card", Recent Developments in Intelligent Systems and Interactive Applications, Lecture Notes in Computer Science, Springer, 2017.

[24] Y. Wang and X. Peng, "Cryptanalysis of Two Efficient Password-based Authentication Schemes Using Smart Cards", International Journal of Network Security, Vol. 17, No. 6, pp. 728-735, 2015.

[25] J. Wei, W. Liu, X. Hu, "Secure and Efficient Smart Card Based Remote User Password Authentication Scheme", International Journal of Network Security, Vol. 18, No. 4, pp. 782$792,2016$.

[26] H. Wijayanto, M. S. Hwang, "Improvement on Timestampbased User Authentication Scheme with Smart Card Lost Attack Resistance", International Journal of Network Security, Vol. 17, No. 2, 2015, pp. 160-164, 2015.

[27] H. Zhu, Y. Zhang, and Y. Zhang, "A Provably Password Authenticated Key Exchange Scheme Based on Chaotic Maps in Different Realm", International Journal of Network Security, Vol. 18, No. 4, pp. 688-698, 2016

[28] X. Zhuang, C. C. Chang, Z. H. Wang, Y. Zhu, "A Simple Password Authentication Scheme Based on Geometric Hashing Function", International Journal of Network Security, Vol. 16, pp. 271-277, 2014

\section{Creative Commons Attribution License 4.0 (Attribution 4.0 International, CC BY 4.0)}

This article is published under the terms of the Creative Commons Attribution License 4.0 https://creativecommons.org/licenses/by/4.0/deed.en_US 